\title{
Second-harmonic imaging of cornea after intrastromal femtosecond laser ablation
}

\author{
Meng Han \\ Leander Zickler \\ University of Heidelberg \\ Kirchhoff Institute for Physics \\ Im Neuenheimer Feld 227 \\ D-69120 Heidelberg \\ Germany \\ E-mail: mhan@urz.uni-heidelberg.de
}

\section{Guenter Giese}

Max-Planck-Institute for Medical Research

Jahnstrasse 29

D-69120 Heidelberg

Germany

\section{Matthias Walter}

University of Heidelberg

Kirchhoff Institute for Physics

Im Neuenheimer Feld 227

D-69120 Heidelberg

Frieder $\mathbf{H}$. Loesel

20/10 Perfect Vision

Am Taubenfeld 21/1

D-69123 Heidelberg

Germany

\section{Josef F. Bille}

University of Heidelberg

Kirchhoff Institute for Physics

Im Neuenheimer Feld 227

D-69120 Heidelberg

\begin{abstract}
Nonlinear laser scanning microscopy is widely used for noninvasive imaging in cell biology and tissue physiology. However, multiphoton fluorescence imaging of dense, transparent connective tissue (e.g., cornea) is challenging since sophisticated labeling or slicing is necessary. High-resolution, high-contrast second harmonic generation (SHG) imaging of corneal tissue based on the intrinsic structure of collagen is discussed. The three-dimensional corneal ultrastructure in depths up to hundreds of microns can be probed noninvasively, without any staining or mechanical slicing. As an important application of second harmonic imaging in ophthalmology, the modification of corneal ultrastructure using femtosecond laser intrastromal ablation is systematically investigated to evaluate nextgeneration refractive surgical approaches. ๑ 2004 Society of Photo-Optical Instrumentation Engineers. [DOI: 10.1117/1.1756919]
\end{abstract}

Keywords: second harmonics; imaging; multiphoton; microscopy; cornea; femtosecond; all-solid-state laser.

Paper 03097 received Jul. 17, 2003; revised manuscript received Oct. 24, 2003; accepted for publication Nov. 12, 2003

\section{Introduction}

\subsection{Neodymium:glass Femtosecond Laser for Ocular Surgery}

In the past several decades, the development of ultrashort pulsed lasers has advanced laser science. Femtosecond (fs) pulsed lasers are powerful tools, not only for fundamental research in nonlinear optics, chemical dynamics, and laser spectrometry, but also for broad applications in fiber communications, nonlinear laser microscopy, micromanipulation of biological systems, and tissue engineering. Owing to its accessibility and transparency, the human eye is a prime candidate for laser-based diagnostic and therapeutic applications. In the past decade, there have been many publications to explore novel applications of ultrafast lasers for refractive surgery. ${ }^{1-6}$

Among the different types of fs laser systems, the all-solidstate neodymium (Nd):glass laser offers system parameters (wavelength, peak fluence, stability, cost) optimally suited for clinical use in ophthalmic surgery. ${ }^{7}$ Since the near-infrared (NIR) wavelength $(\lambda=1.06 \mu \mathrm{m})$ allows high transmission through corneal tissue, the concept of intrastromal or noninvasive refractive surgeries will be feasible. ${ }^{8}$ Although the single NIR photon energy is not high enough to break cova-

Address all correspondence to: Dr. Meng Han, University of Heidelberg, Kirchhoff Institut for Physik, INF227, D-69120 Heidelberg, Germany; Tel: 496221 549254; Fax: 496221 549112; E-mail: mhan@urz.uni-heidelberg.de lence chemical bonds in ocular tissue, multiphoton absorption is triggered by the extremely high laser intensity in the focal region. Free carriers are produced nearly independently of intrinsic defects or incursions, resulting in a repeatable and predictable ablation outcome. The subsequent avalanche ionization leads to laser-induced optical breakdown (LIOB) and the formation of a microplasma. ${ }^{9-14}$ The rapid expansion of the laser plasma and a propagating shock wave efficiently dissociate the dense corneal tissue. Owing to the ultrashort time scale, the absorbed laser energy is highly localized. Side effects and thermal damage are greatly reduced compared with nanosecond (ns) excimer lasers or picosecond (ps) lasers. Since the threshold fluence for LIOB approximately shows a square root dependence on the pulse duration, ${ }^{15-18}$ ultrashort pulse lasers minimize the total energy deposited into the tissue.

It is also worth mentioning that NIR fs laser surgery minimizes the risk of mutagenicity and toxicity possibly encountered with UV irradiation by excimer lasers. The $\mathrm{TEM}_{00}$ mode of an all-solid-state fs laser enables almost diffraction-limited focusing, providing a beam quality much better than the multimode excimer laser. In summary, the all-solid-state diodepumped Nd:glass fs laser meets all key parameters for a minimally invasive surgical laser system in ophthalmology.

$1083-3668 / 2004 / \$ 15.00$ @ 2004 SPIE 


\subsection{Nonlinear Laser Scanning Microscopy}

The development of ultrafast lasers enabled the discovery of a novel class of optical microscopes-the nonlinear laser scanning microscope. ${ }^{19}$ To form a microscopic image, the signal collected in a nonlinear laser microscope does not originate from the reflection or refraction of the illuminating light beam, but from nonlinear optical processes induced by the ultrahigh photon flux, such as multiphoton absorption, multiharmonic generation, four-wave mixing, or coherent Raman scattering. ${ }^{20}$ Compared with traditional optical microscopes, the nonlinear laser scanning microscope offers a number of advantages: (1) Since multiphoton absorption can be confined to the region of peak intensity within the focus of the illuminating laser beam, diffraction-limited resolution and depth discrimination are achievable without extra pinholes, compared with confocal microscopy. (2) The photodamage or bleaching effect outside the laser focus is greatly reduced, which is crucial in imaging living cells. However, in the focal plane, the photodamage can increase with a high power (on the order of 3) of the excitation intensity. ${ }^{21,22}$ (3) Infrared lasers can be utilized to achieve both a deeper sensing depth and less photodamage than ultraviolet or blue light excitation, which are typically used in single-photon fluorescence microscopy.

Several implementations of nonlinear laser scanning microscopy have been developed, namely, multiphoton excited fluorescence microscopy, multiharmonics microscopy, and coherent anti-Stokes Raman scattering microscopy (CARS). Owing to the rich resource of fluorescent dyes available for selective and nontoxic staining and innovative recording techniques such as fluorescence resonant energy transfer (FRET) and fluorescence recovery after photobleaching (FRAP), multiphoton excited fluorescence microscopy is widely applied in imaging living cells, probing single molecules, and the investigation of biochemical processes.

\subsection{Motivations and Research Questions}

Prior to the clinical application of fs surgical lasers, a highresolution microscopic analysis of the target tissue is necessary to investigate the precision and efficiency of the new surgical strategy, to evaluate side effects of fs laser surgery, or to monitor the tissue healing process after laser surgery. The ultrastructure of corneal tissue must be characterized under conditions closest to its natural physiological state. Previous studies utilized scanning-transmission electron microscopy or other standard methods of histological analysis. ${ }^{23,24}$ The required fixation, embedding, and slicing procedures can produce considerable artifacts during the sample preparation process, and are tedious and time-consuming. Therefore, a noninvasive, high-resolution all-optical imaging method would be highly rewarding in characterizing corneal ultrastructure and evaluating fs laser intrastromal surgery effects.

Multiphoton excitation fluorescence microscopy is outstanding for noninvasive imaging by largely preventing damage of the specimen during investigation. There has been a rapidly growing number of applications, particularly in the field of cellular and molecular biology. Cells can be imaged in aqueous buffer solutions similar to their natural environment. But for imaging corneal tissue, the situation is quite different. In order to achieve satisfactory image contrast from multipho- ton fluorescence, fluorescent labeling of the corneal tissue is required, but this is difficult to achieve. This article addresses the following question: Is it possible to use a multiphoton microscopy technique for in-depth imaging of corneal tissue without staining or slicing? The image contrast should arise from the intrinsic properties of the tissue component. As we can show in this article, collagen as a major component of corneal tissue can meet the requirements.

The basic structure of collagen is a triple helix composed of three protein chains. Each chain is formed by a repeated sequence of three amino acids. Such structures lack a center of symmetry, which gives collagen the intrinsic ability of second harmonic generation (SHG). A detailed description of SHG from collagen and other biological systems has been given in previous publications. ${ }^{25-28}$ As a second-order nonlinear excited process, the SHG signal increases with the square of excitation intensity. The same relationship holds for twophoton excited fluorescence. Thus the features of two-photon fluorescence imaging such as deep sensing depth and diffraction-limited resolution are inherited with SHG imaging. ${ }^{34,35}$

It is worthwhile to take a brief look at the history of frequency conversion. SHG from crystals and interfaces was first independently discovered by Kleinman and Bloembergen. ${ }^{29,30}$ It has found many applications in frequency doubling and spectroscopic analysis of interface dynamics. The first quantitative study of SHG from collagen was pioneered by Roth and Freund. ${ }^{25,31,32}$ They used SHG to study the orientation of collagen fibers in rat tail tendon with a resolution of $50 \mu \mathrm{m}$. Only recently, after Gauderon, Campagnola, and Cox combined SHG imaging, an ultrafast pulsed laser source, and modern nonlinear laser scanning microscopy techniques could, ${ }^{26,27,33,34}$ the advantages of SHG microscopy be fully appreciated. The resolution is now comparable to that of confocal or multiphoton fluorescence microscopy. Owing to the simplicity of the SHG imaging setup, it attracts increasing attention from the microscopy community. There has been much interesting work ${ }^{36-38}$ toward novel applications and fundamental studies of second harmonic excitation and imaging. To the best of our knowledge, our group is the first ${ }^{6}$ to report on the systematic evaluation of femtosecond laser intrastromal surgery results using SHG imaging of corneal ultrastructure.

\section{Materials and Methods}

\subsection{Porcine Cornea Preparation}

An excised porcine eye was obtained from the local slaughterhouse. After fs laser treatment, the porcine eye was kept moist with $4 \%$ phosphate-buffered saline (PBS, $\mathrm{pH}$ 7.4) drops until the cornea regained transparency (about $30 \mathrm{~min}$ ). Then the cornea was excised and fixed with freshly prepared paraformaldehyde (4\% in $\mathrm{PBS}, \mathrm{pH} 7.4$ ).

All porcine cornea samples were surgically treated by a diode-pumped mode-locked Nd:glass laser $(\lambda=1.06 \mu \mathrm{m}, \tau$ $=700 \mathrm{fs}, E=5 \sim 10 \mu \mathrm{J}, f=4 \sim 12.5 \mathrm{kHz}$ ). The laser beam was guided to the porcine eye through a lens pair with a variable focal length (Z-scan) and two galvanometer mirrors enabling a rapid XY-scan. The laser spot diameter in the focal plane was $5 \mu \mathrm{m}$. 


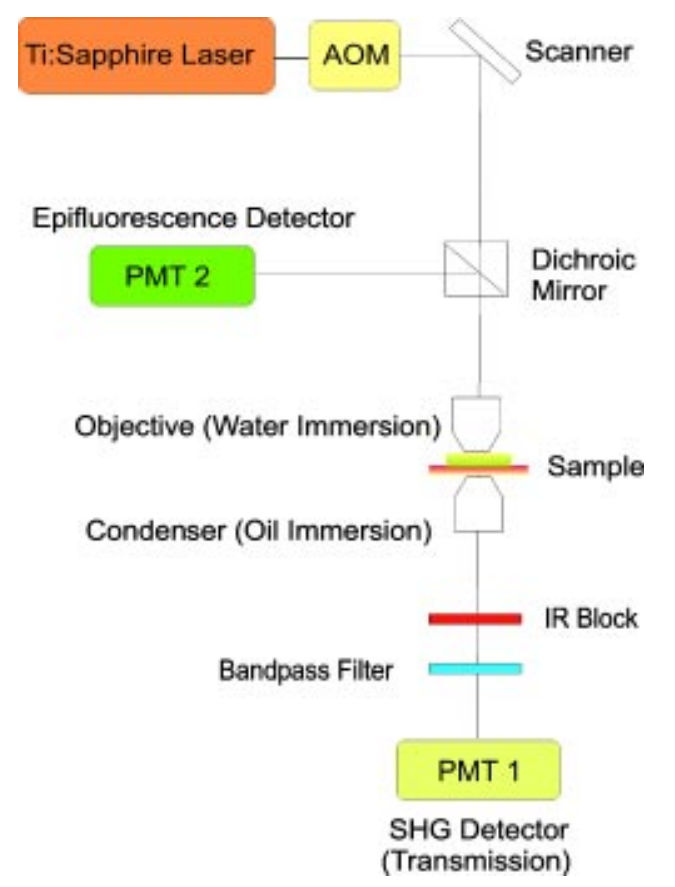

Fig. 1 Conceptual drawing of an SHG laser scanning microscope.

Various laser pulse sequences were applied to the porcine cornea, covering the range from single-pulse intrastromal ablation (individual cavitation bubble), corneal ring and flap cutting (two-dimensional collective effect) to an intrastromal laser-ablated cavity (three-dimensional laser-tissue interaction). The cornea samples presented here received three types of laser treatment: (1) Using a circular laser scan with a spot spacing larger than the typical cavitation bubble size, which is around $50 \mu \mathrm{m}$, the individual effect of a pulsed laser-tissue interaction is investigated. (2) Using a cylindrical scan with a diameter of $10 \mathrm{~mm}$ and a spot spacing of $12 \mu \mathrm{m}$, a corneal ring cutting is completed to investigate the collective effect of laser intrastromal ablation. (3) By applying a spiral scanning pattern with a fixed depth of $150 \mu \mathrm{m}$, an intrastromal corneal flap bed is created. Detailed descriptions of similar laser treatment procedures can be found in previous publications. ${ }^{1,3,4}$

\subsection{SHG Imaging Experimental Setup}

The SHG imaging was performed on a Zeiss LSM 510 NLO laser scanning multiphoton microscope (Zeiss, Jena, Germany) with minor modifications (as illustrated in Fig. 1). The excitation laser source was a mode-locked titanium:sapphire laser (Ti:S) (Coherent Mira Fs, Coherent Inc., Santa Clara, California), tunable from 700 to $1000 \mathrm{~nm}$, pumped by a solidstate laser (Verdi, $8 \mathrm{~W}$, Coherent Inc.). In this study, the Ti:S laser emission wavelength was set to $880 \mathrm{~nm}$. The laser intensity was attenuated using an acoustic optic modulator (AOM Zeiss). The acquisition of a single $512 \times 512$-pixel image was generally achieved in a few seconds (fast laser scan with galvanometer scanners) and a typical image stack of porcine cornea could be finished within 20 min ( $Z$ stack size $\approx 1.5 \mathrm{~mm}, \mathrm{Z}$ step size: $5 \mu \mathrm{m})$. A $40 \times / 0.8$ numerical aperture (NA) water-immersion objective was used for high-resolution imaging of the cornea sample. Owing to the coherent generation of the second harmonic signal from bulk collagen, the
Frequency Conversion Exponent $\kappa=2.003 \pm 0.038$

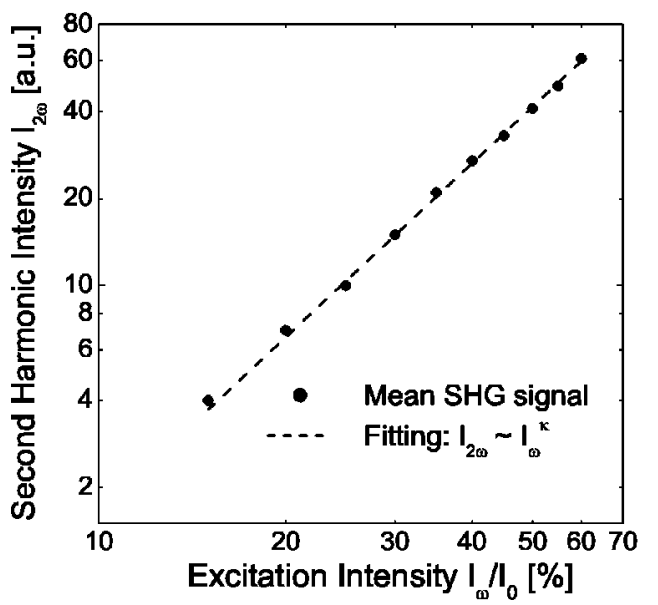

Fig. 2 Average second harmonic intensity $I_{2 \omega}$ measured in transmission as a function of normalized illumination intensity $I_{\omega} / I_{0}\left(I_{0}\right.$; maximal Ti:S laser output intensity). A least-squares fit (dashed line) is in perfect agreement with our theory; the measured exponent of SHG frequency conversion is 2.003 .

signal is emitted predominantly in the transmission direction. $^{20,21}$ Therefore, a 1.4 NA oil immersion condenser was employed to efficiently collect the SHG signal. Two IR beam block filters in sequence (Zeiss KP685) and a narrow bandpass filter $(440 / 10 \mathrm{~nm})$ in front of the transmission light path photomultiplier tube (PMT) ensured that illumination light was excluded and only second harmonic signals from the corneal tissue were recorded.

\section{Microscopic Results}

\subsection{Verification of SHG from Collagen}

A number of results verified that the signal detected in transmission represented SHG from collagen: (1) Intensity dependence: Using intensity modulation, we tuned the Ti:S laser output power to investigate the influence of the excitation beam's intensity on SHG. In an otherwise unprocessed cornea sample, a $230 \times 230-\mu \mathrm{m}$ frame was scanned with the Ti:S laser. Using a scan rather than a fixed beam avoids photobleaching effects. Using image analysis, an average second harmonic intensity and signal standard deviation (contrast) was extracted. The relationship between the excitation laser power and the mean SHG intensity is illustrated in Fig. 2. Through least-squares fitting, a perfect quadratic intensity dependence of SHG could be confirmed. (2) Line spectrum emission: Only when using a narrow bandpass filter that exactly matched half of the excitation wavelength $(\lambda / 2$ $=440 \mathrm{~nm}$ ), were strong signals detected by the PMT detector. Shifting the central wavelength of the filter by only $10 \mathrm{~nm}$ $(450 / 10 \mathrm{~nm})$ completely blocked the observed signal. With the latter filter, high-contrast signals could again be obtained by tuning the Ti:S laser wavelength to $900 \mathrm{~nm}$. The bandpass filter experiments excluded a possible two-photon excited autofluorescence, which typically has a relatively broad emission spectrum. (3) Coherent excitation: We observed that the second harmonic signals were detected (as theoretically predicted) predominantly in the forward direction, with only a very weak signal in the epi-fluorescence light path, which 


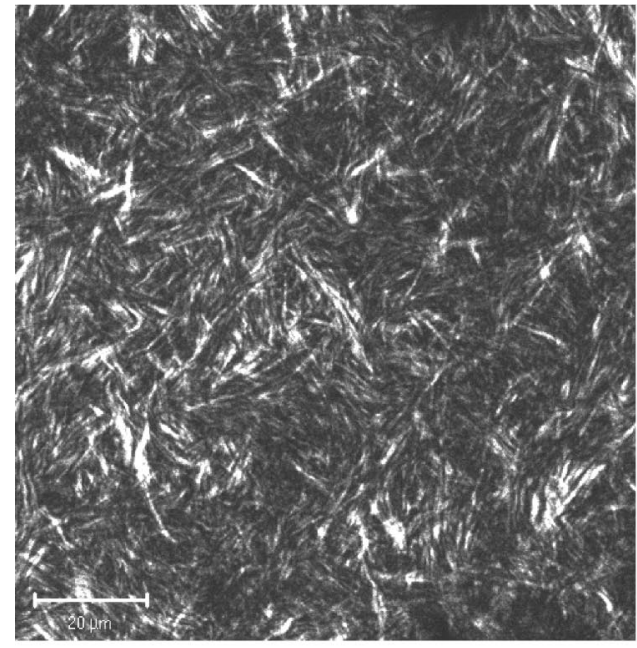

(a)

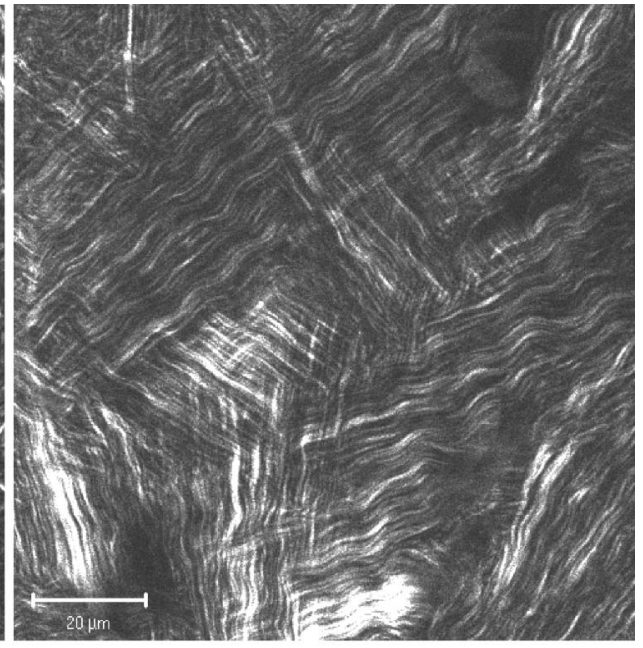

(b)

Fig. $3 \mathrm{SHG}$ optical sections from (a) the corneal surface, depth=10 $\mu \mathrm{m}$ and (b) from the corneal stroma, depth=200 $\mu \mathrm{m}$. Bar=20 $\mu \mathrm{m}$.

mostly probably originated from scattered SHG signals. (4) Nonlinear process: Frequency conversion does not take place when running the Ti:S laser in continuous wave (cw) mode, indicating that the observed signal corresponds to a nonlinear excitation requiring high peak intensities. In summary, a number of results independently verify that the high-contrast signals measured in transmission are second harmonics signals generated by corneal collagen.

As illustrated in Fig. 3, the structure of the corneal collagen fiber is clearly revealed by SHG imaging. In correlation with the $Z$-position of the scan, two characteristic collagen fiber distributions were observed. In the surface region (first $10 \mu \mathrm{m})$, the collagen fibers were short, small, and randomly arranged. At a depth of $200 \mu \mathrm{m}$, the collagen fibers demonstrated remarkable regularity and characteristic undulation. They were densely packed, highly ordered, and ran parallel to the corneal surface. Most collagen fibers shared the orientation with their neighbors, except that in few places the adjacent collagen fibers were at right angles. Figure 3 provides direct evidence for the reliability of SHG imaging methods in studying the cornea, since the SHG image agreed perfectly with the well-known histological characteristics of the cornea. ${ }^{39}$ The anterior Bowman's layer (10 $\mu \mathrm{m}$ thick) consists of randomly distributed collagen fibers. The corneal stroma is a dense connective tissue composed of flattened collagenous lamellae ( $2 \mu \mathrm{m}$ thick). Collagenous lamellae are highly organized collagen fibers that are arranged in orientation domains. Adjacent domains have an almost perpendicular fiber orientation.

As shown in Fig. 3, SHG imaging is outstanding in revealing the distribution and orientation of collagen fibers, even if they are located at depths of hundreds of microns. The technique does not involve any sectioning or staining of the volume under investigation. This is particularly important for the noninvasive evaluation of laser intrastromal surgery. The cornea can now be investigated under conditions very close to its physiological state, without possible complications and artifacts induced by additional sample preparation or slicing processes. With typical excitation laser intensities for SHG im- aging, we observed that even for long measurement durations (high-resolution stack data), there is no noticeable photobleaching effect. We also compared SHG images from fresh porcine cornea with an old cornea sample stored in formalin for over 2 months and found that the detected SHG intensity was comparable. In conclusion, our study of virgin porcine cornea proves that SHG imaging is a noninvasive, repeatable, and well-suited imaging technique for analyzing corneal ultrastructure.

\subsection{SHG Imaging of Corneal Ultrastructure}

Although the interaction between ultrafast laser radiation and corneal tissue is a complicated process that includes multiphoton absorption, cascade ionization, self-focusing, plasma absorption or shielding, and shock wave production, the lasting laser surgery outcome is determined mainly by two factors: tissue removal by to laser ablation and tissue deformation that is due to plasma expansion and shock wave propagation. Ablation of material is supposed to directly influence the thickness and curvature of the cornea. This modifies the contribution of the cornea to the eye's refraction and determines the precision of laser intrastromal surgery. Expansion of the laser plasma also affects the location and orientation of collagen fibers and in turn the optical properties of the cornea. Here we focus on the boundary region of the intrastromal structures produced by fs laser ablations, where the image contrast is extremely high owing to loss of SHG in the laser-produced cavity. Then we will evaluate the efficacy and precision of laser surgery by characterizing the size and geometry of the induced intrastromal cavities. The side effects can be nicely analyzed by visualization of the distribution and orientation of the adjacent corneal tissue.

Our simplest case of laser-tissue interaction is intrastromal ablation by a single laser pulse. The effect of single-pulse irradiation is illustrated in Fig. 4, where the pulse energy was set to be well above the cornea ablation threshold to produce a cavitation bubble inside the corneal stroma. After laser irradiation, the laser plasma-induced cavitation bubble and its 


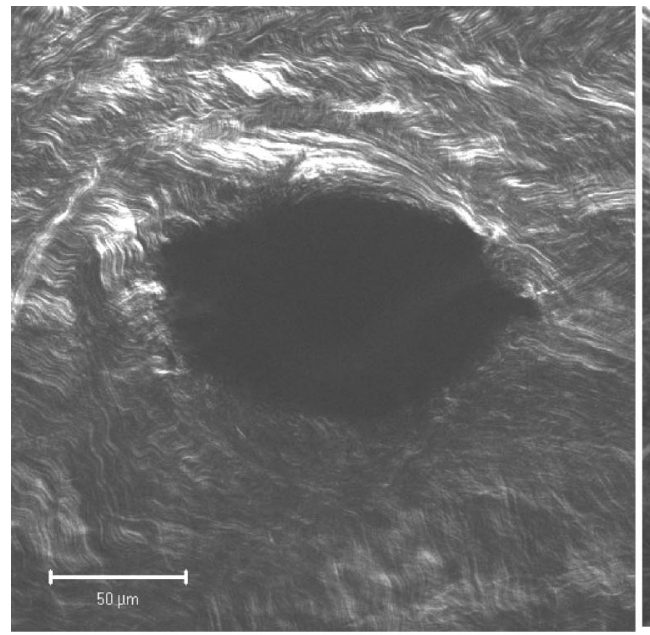

(a)

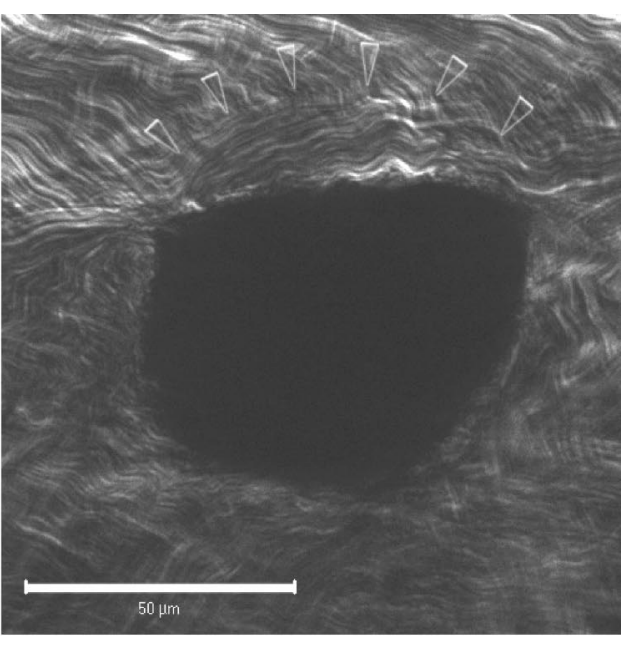

(b)

Fig. 4 SHG imaging of two cavitation bubbles each induced by a single-pulse intrastromal fs laser ablation. Bar $=50 \mu \mathrm{m}$.

border region, including the deformation and reorientation of the adjacent collagen fibers, can be clearly recognized (Fig. 4). The collagen fibers surrounding the cavitation bubble were nearly untouched; there is no indication of thermal damage. Figure 4(b) shows an interesting cavitation bubble deviating from the normal elliptical shape [Fig. 4(a)]. In the upper area of the cavitation bubble, a smooth elliptical boundary can still be recognized (indicated by the open arrowheads), which could correspond to the original shape of the cavitation bubble. We suppose that after the cavitation bubble collapsed, collagen fibers partially regained their initial location and orientation. This could be beneficial for the wound-healing process, since less tissue damage seems to be involved.

To investigate the collective effects of laser pulse sequences, a ring cutting pattern was applied to the cornea sample by continuously incrementing the laser focus $\mathrm{Z}$ position during the circular scan. An optical section of the laser cutting ring is shown in Fig. 5. The cutting borders on both sides are very smooth. Adjacent to the cutting interfaces, most collagen fibers appear to have preserved their original distribution and orientation. The plasma and shock wave-damaged zone is confined within micrometers, as indicated by the agglomeration of collagen fibers (indicated by arrowheads). The constant cutting width confirms the repeatable and deterministic nature of fs laser intrastromal ablations, which is extremely important for a predictable surgical outcome. As for clinical applications, corneal keratoplasty can directly benefit from its high precision and minimal side effects. Irregularly shaped donor corneas may now be obtained with complete design flexibility. Novel graft geometries require fewer sutures (possibly none at all) and promise better visual quality for the patient.

The corneal cutting ring demonstrates the excellent vertical ablation quality of the fs surgical laser. In order to evaluate the lateral ablation performance, the laser focus was fixed to $150 \mu \mathrm{m}$ beneath the surface. A spiral scanning pattern was applied in the $x, y$-plane to produce an intrastromal cavity. After surgical laser treatment, the cornea sample was kept unsliced and unstained, and a three-dimensional image stack was obtained by optical sectioning. As shown in Fig. 6, lateral surgical laser spot scanning produced an intrastromal cavity in the focal plane. The anterior and posterior surfaces of the intrastromal cavity are well separated. From our experience, the flap could be easily opened without special skill or training.

The all-optical flap-cutting procedure is another application of fs lasers in refractive surgery and laser in situ keratomileusis (LASIK). In LASIK, the corneal flap is traditionally cut with a mechanical microkeratome. The surgeon's experience determines the flap thickness, and reproducible results are difficult to obtain. With the fs laser, the thickness and location of the flap can be precisely controlled by the 3-D focus control of the application unit. ${ }^{1}$

Besides their potential applications in keratoplasty and LASIK, fs lasers also offer a novel possibility of noninvasive intrastromal correction of vision. The basic idea is to apply

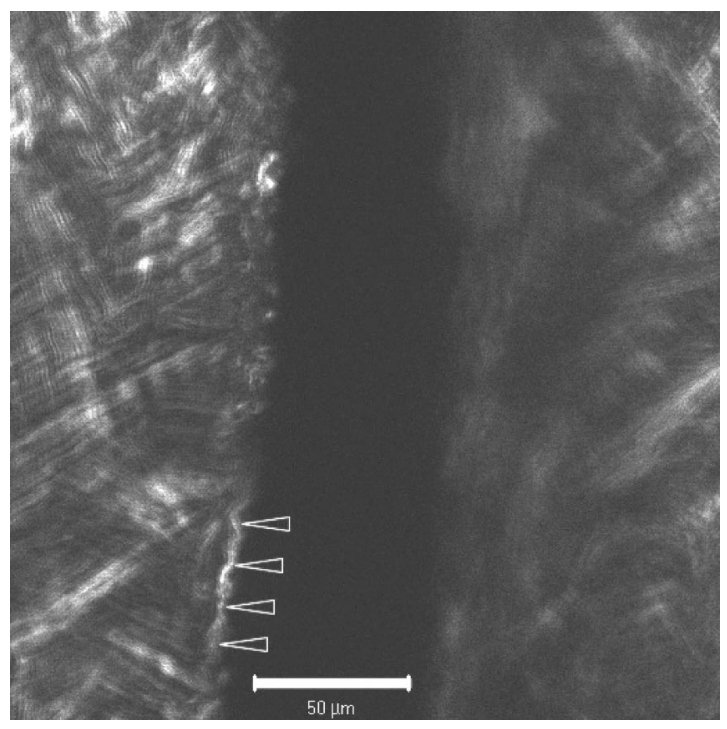

Fig. 5 SHG optical XY-section of a cornea cutting ring. The ring pattern is generated by a cylindrical scanning of the $\mathrm{Nd}$ :glass fs laser. The tissue damage region is indicated by the arrowheads. Bar $=50 \mu \mathrm{m}$. 


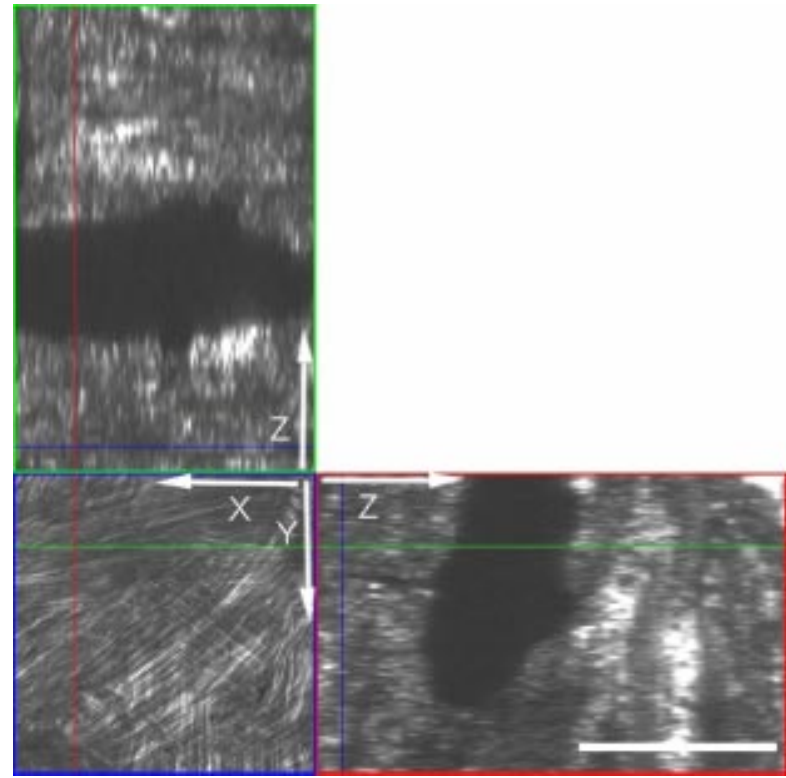

Fig. 6 Three-dimensional SHG stack image of cornea structures modified by intrastromal fs laser ablation. Bar $=40 \mu \mathrm{m}$. In anticlockwise sequence, the images correspond to $\mathrm{XZ}, \mathrm{XY}$, and $\mathrm{YZ}$ sections, respectively.

several different intrastromal ablation patterns at different depths to selectively remove corneal tissue. During laser irradiation, the cornea is opacified, owing to the formation of cavitation bubbles, where the corneal stroma is transformed to gas and vapors. The gas or vapor then diffuses out of the cornea or is resorbed within hours, and the cornea regains its transparency. As a consequence, the refractive properties of the cornea are modified by the removal of corneal tissue and collagen deformation. Since no opening of the cornea and no mechanical processing is involved, corneal damage is largely reduced. Compared with the excimer laser procedure, noninvasive intrastromal ablation strategy by a fs laser could be valuable for corrections of early-stage vision aberrations (e.g., for young patients) and post-LASIK or photo refractive keratectomy (PRK) touchups.

\section{Discussion and Conclusion}

It is difficult to probe corneal tissue structure by two-photon fluorescence microscopy. However, based on its noncentrosymmetric structure, collagen, the major component of corneal tissue, has the unique and intrinsic ability to frequency convert an incoming ultrashort laser pulse to its second harmonic. This gives us the chance to probe the collagen distribution in the surgically treated cornea with noninvasive SHG microscopy. Owing to the nonlinear two-photon absorption process, a pulsed IR laser can be used for SHG excitation, allowing imaging deep into the corneal tissue. The intensity of the second harmonic signal is quadratically dependent on the incident laser intensity; thus a micrometer spatial resolution is achieved without pinholes. Thick, dense, and unfixed corneal tissue can be easily imaged with strong image contrast. Both the resolution and sensing depth of SHG microscopy are com- parable to two-photon fluorescence microscopy. At the same time, the method does not require any staining or mechanical slicing.

With a series of experimental verifications, notably the quadratic dependance between the second harmonic and the fundamental as well as its narrow linewidth, and the characteristic signal pattern, we confirmed that the signals detected in the transmission direction correspond to second-order frequency conversion by corneal collagen. The cornea samples maintained their SHG ability throughout months of storage. Images obtained by SHG imaging agree well with known histological findings. Large sensing depth and high resolution make SHG microscopy an outstanding tool for the noninvasive imaging of corneal ultrastructure. As a consequence, SHG imaging was utilized to evaluate the efficacy and collateral effects of mini-invasive femtosecond laser surgical strategies. The laser-induced intrastromal cavity can be noninvasively visualized with strong image contrast and high resolution. By analyzing the reorientation or deformation of the adjacent collagen fibers, the tissue damage can be traced with high sensitivity. Both the individual and collective effects of intrastromal fs laser ablations have demonstrated a high surgical precision with minimal side effects.

However, biological systems are dynamic systems. Even if immediately after laser surgery the surgical outcome agrees with the expected results, the long-term effect may be quite different. Regarding tissue healing and regeneration issues, an in vivo or time-resolved study is crucial for future investigations. Based on the intrinsic structure of collagen, second harmonic imaging can be one of the most suitable methods for tracing the tissue-healing process after intrastromal laser surgery. Although putting a detector on the far side of the cornea might be impractical, probably there are sufficient reflected SHG signals from the highly reflective retinal pigment epithelium layer for evaluation.

It is worth mentioning that the experimental setup for SHG imaging is rather simple. Only minor changes of the nonlinear multiphoton fluorescence setup are required to perform SHG imaging. Since collagen is the most abundant protein in the human body, in addition to ophthalmology, SHG imaging of collagen may lead to other potential applications in medical research, including the microscopic anatomy of connective tissue or new noninvasive diagnostic methods.

\section{Acknowledgments}

The authors wish to thank Martin Weinacht and Ralf Kessler from 20/10 Perfect Vision for expertly running the fs surgical laser. We are indebted to Winfried Denk for access to the microscopy facility of the Max-Planck Institute for Medical Research, Heidelberg, and to Heinz Horstmann for corneal sample preparations. This work was partially supported by the BMBF Femtosecond Technology (FST) project.

\section{References}

1. J. F. Bille, C. F. H. Harner, and F. Loesel, Eds., New Frontiers in Vision and Aberration-Free Refractive Surgery, Springer-Verlag, Heidelberg (2002).

2. M. H. Niemz, Laser-Tissue Interactions: Fundamentals and Applications, Springer-Verlag, Berlin (1996).

3. R. M. Kurtz, C. Horvath, H. H. Liu, R. R. Krueger, and T. Juhasz, "Lamellar refractive surgery with scanned intrastromal picosecond 
and femtosecond laser pulses in animal eyes," J. Refract. Surg. 14, 541 (1992).

4. T. Juhasz, F. H. Loesel, C. Horvath, R. M. Kurtz, and G. Mourou, "Corneal refractive surgery with femtosecond lasers," IEEE J. Sel. Top. Quantum Electron. 5, 902-910 (1999).

5. F. H. Loesel, J. P. Fischer, M. H. Goetz, C. Horvath, T. Juhasz, F. Noack, N. Suhm, and J. F. Bille, "Non-thermal ablation of neural tissue with femtosecond laser pulses," Appl. Phys. B: Lasers Opt. 66, 121-128 (1998).

6. L. Zickler, M. Han, G. Giese, F. H. Loesel, and J. F. Bille, "Femtosecond all-solid-state laser for refractive surgery," Proc. SPIE 4978, 194-207 (2003).

7. L. Zickler, "Femtosecond laser for highly precise ophthalmic surgery and novel imaging method of corneal ultrastructure modifications," $\mathrm{PhD}$ dissertation, University of Heidelberg, 2003.

8. M. H. Niemz, T. Hoppeler, T. Juhasz, and J. F. Bille, "Intrastromal ablations for refractive corneal surgery using picosecond infrared laser pulses," Laser Light Ophthalmol. 5, 149-155 (1993).

9. J. G. Fujimoto, W. Z. Lin, E. P. Ippen, C. A. Puliato, and R. F. Steinert, "Time-resolved studies of Nd:YAG laser-induced breakdown," Invest. Ophthalmol. Visual Sci. 26, 1771-1777 (1985).

10. N. Bloembergen, "Laser-induced electric breakdown in solids," IEEE J. Quantum Electron. 10, 375-386 (1974).

11. P. K. Kennedy, S. A. Boppart, D. X. Hammer, B. A. Rockwell, G. D. Noojin, and W. P. Roach, "A first-order model for computation of laser-induced breakdown thresholds in ocular and aqueous media: Part I Theory and Part II Comparison to Experiment," IEEE J. Quantum Electron. 31, 2241-2257 (1995).

12. D. X. Hammer, R. J. Thomas, G. D. Noojin, B. A. Rockwell, P. K. Kennedy, and W. P. Roach, "Experimental investigation of ultrashort pulse laser-induced breakdown thresholds in aqueous media," IEEE J. Quantum Electron. 32, 670-678 (1996).

13. A. Vogel, P. Schweiger, A. Freiser, M. N. Asyo, and R. Birngruber, "Intraocular Nd:YAG laser surgery: light-tissue interactions, damage range, and reduction of collateral effects," IEEE J. Quantum Electron. 26, 2240-2260 (1990).

14. A. Vogel, W. Hentschel, J. Holzfuss, and W. Lauterborn, "Cavitation bubble dynamics and acoustic transient generation in ocular surgery with pulsed neodymium: YAG laser," Ophthalmology 93, 1259-1269 (1986).

15. F. H. Loesel, M. H. Niemz, J. F. Bille, and T. Juhasz, "Laser-induced optical breakdown on hard and soft tissues and its dependence on the pulse duration: experiment and model," IEEE J. Quantum Electron. 32, 1717-1722 (1996).

16. F. H. Loesel, A. C. Tien, S. Backus, H. Kapteyn, M. Murnane, R. M. Kurtz, S. Sayegh, and T. Juhasz, "Effect of reduction of laser pulse width from 100 ps to $20 \mathrm{fs}$ on the plasma-mediated ablation of hard and soft tissue," Proc. SPIE 3565, 116-123 (1999).

17. R. M. Kurtz, X. Liu, V. M. Elner, J. A. Squier, D. Du, and G. A. Mourou, "Plasma-mediated ablation in human cornea as a function of laser pulse width," J. Refract. Surg. 13, 653-658 (1997).

18. D. Du, J. Squier, R. M. Kurtz, V. Elner, X. Liu, G. Gutmann, and G. Mourou, "Damage threshold as a function of pulse duration in biological tissue," in Ultrafast Phenomena Vol. IX, P. F. Barbaraetal, Ed., pp. 254-255, Springer-Verlag, Berlin (1995).

19. W. Denk, J. H. Strickler, and W. W. Webb, "Two-photon laser scanning fluorescence microscopy," Science 248, 73-76 (1990).

20. J. X. Cheng, Y. K. Jia, G. F. Zheng, and X. S. Xie, "Laser-scanning coherent anti-Stokes Raman scattering microscopy and applications to cell biology," Biophys. J. 83, 502-509 (2002).
21. G. H. Patterson and D. W. Piston, "Photobleaching in two-photon excitation microscopy," Biophys. J. 78, 2159-2162 (2000).

22. G. Chirico, F. Cannone, G. Baldini, and A. Diaspro, "Two-photon thermal bleaching of single fluorescent molecules," Biophys. J. 84, 588-598 (2003).

23. G. Maatz, A. Heisterkamp, H. Lubatschowski, S. Barcikowski, C. Fallnich, H. Welling, and W. Ertmer, "Chemical and physical side effects at application of ultrashort laser pulses for intrastromal refractive surgery," J. Opt. A2, 59-64 (2000).

24. H. Lubatschowski, A. Heisterkamp, F. Will, J. Serbin, T. Bauer, C. Fallnich, and H. Welling, "Ultrafast laser pulses for medical applications," Proc. SPIE 4633, 38-49 (2002).

25. I. Freund, M. Deutsch, and A. Sprecher, "Connective tissue polarity, optical second-harmonic microscopy, crossed-beam summation, and small-angle scattering in rat-tail tendon," Biophys. J. 50, 693-712 (1986).

26. P. J. Campagnola, H. A. Clark, W. A. Mohler, A. Lewis, and L. M. Loew, "Second-harmonic imaging microscopy of living cells," $J$. Biomed. Opt. 6, 277-286 (2001).

27. G. Cox, E. Kable, A. Jones, I. Fraser, F. Manconi, and M. D. Gorrell, "3-Dimensional imaging of collagen using second harmonic generation," J. Struct. Biol. 141, 53-62 (2003).

28. R. Gauderon, P. B. Lukins, and C. J. R. Sheppard, "Optimization of second harmonic generation microscopy," Micron 32, 691-700 (2002).

29. D. A. Kleinman, A. Ashkin, and G. D. Boyd, "The second harmonic generation of light by focused laser beams," IEEE J. Quantum Electron. 2, 425-429 (1966).

30. N. Bolembergen, R. K. Chang, S. S. Jha, and C. H. Lee, "Optical second-harmonic generation in reflection from media with inversion symmetry," Phys. Rev. 174, 19813-19812 (1968).

31. S. Roth and I. Freund, "Second harmonic generation in collagen," $J$. Chem. Phys. 70, 1637-1643 (1979).

32. S. Roth and I. Freund, "Coherent optical harmonic generation in rat-tail," Opt. Commun. 33, 292-296 (1980).

33. R. Gauderon, P. B. Lukins, and C. J. R. Sheppard, "Simultaneous multi-channel nonlinear imaging: combined two-photon excited fluorescence and second harmonic generation microscopy," Micron 32, $685-689$ (2001).

34. P. J. Campagnola, A. C. Millard, M. Terasaki, P. E. Hoppe, C. Malone, and W. A. Mohler, "Three-dimensional high-resolution secondharmonic generation imaging of endogenous structural proteins in biological tissues," Biophys. J. 81, 493-508 (2001).

35. P. J. Campagnola, M. D. Wei, A. Lewis, and L. M. Loew, "Highresolution nonlinear optical imaging of living cells by second harmonic generation," Biophys. J. 77, 3341-3349 (1999).

36. L. Moreaux, O. Sandre, S. Charpak, M. Blanchard-Desce, and J. Mertz, "Coherent scattering in multi-harmonic light microscopy," Biophys. J. 80, 1568-1574 (2001).

37. P. Stoller, K. M. Reiser, P. M. Celliers, and A. M. Rubenchik, "Polarization-modulated second harmonic generation in collagen," Biophys. J. 82, 3330-3342 (2002).

38. X. Y. Deng, E. D. Williams, E. W. Thompson, X. Gan, and M. Gu, "Second-harmonic generation from biological tissues: effect of excitation wavelength," Scanning 24, 175-178 (2002).

39. R. A. Moses and W. M. Hart, Eds., Adlers Physiology of the EyeClinical Application, C. V. Mosby, St. Louis (1987). 